\author{
MITSUBISHI ELECTRIC RESEARCH LABORATORIES \\ http://www.merl.com
}

\title{
Hot-Spot Wireless Access Exploiting Shadowing Diversity of Distributed Antennas
}

\author{
Ye Li, Jinyun Zhang
}

TR2008-051 August 2008

\begin{abstract}
In this paper, we investigate throughput improvement of hot-spot wireless access with distributed antennas. To exploit shadowing diversity of multiple distributed antennas, we propose an antenna selection, which takes both desired signal strength and interference strength into consideration. We also develop a power allocation approach to further enhance the throughput of wireless access. We then use wireless stadium as an example to demonstrate the effectiveness of the proposed approaches.
\end{abstract}

This work may not be copied or reproduced in whole or in part for any commercial purpose. Permission to copy in whole or in part without payment of fee is granted for nonprofit educational and research purposes provided that all such whole or partial copies include the following: a notice that such copying is by permission of Mitsubishi Electric Research Laboratories, Inc.; an acknowledgment of the authors and individual contributions to the work; and all applicable portions of the copyright notice. Copying, reproduction, or republishing for any other purpose shall require a license with payment of fee to Mitsubishi Electric Research Laboratories, Inc. All rights reserved. 



\title{
Hot-Spot Wireless Access Exploiting Shadowing Diversity of Distributed Antennas
}

\author{
Ye (Geoffrey) $\mathrm{Li}^{*}$ \\ School of ECE, Georgia Institute of Technology \\ Jinyun Zhang \\ Mitsubishi Electric Research Labs
}

\begin{abstract}
In this paper, we investigate throughput improvement of hot-spot wireless access with distributed antennas. To exploit shadowing diversity of multiple distributed antennas, we propose an antenna selection approach, which takes both desired signal strength and interference strength into consideration. We also develop a power allocation approach to further enhance the throughput of wireless access. We then use wireless stadium as an example to demonstrate the effectiveness of the proposed approaches.
\end{abstract}

Key Words: Coverage, Signal Processing for Transmission, Modulation \& Multiplexing.

\section{INTRODUCTION}

Hot-spot wireless access is required by more and more applications. One of the critical characteristics of hot-spot wireless access is that there are a lot of mobile users crowded in a small area while the available wireless spectrum is limited.

To improve the throughput of indoor wireless access, distributed antenna techniques have been proposed in [1] - [6] over ten years ago. Recently, it has taken on renewed interesting. In this paper, we will develop an optimal antenna selection approach, which takes both desired signal strength and interference strength into consideration when selecting antennas. We will also propose a pseudo-capacity based power allocation approach.

The rest of the paper is organized as follows. In Section II, we will first introduce wireless propagation model used in our study and then describe wireless access for a stadium. Then in Sections III

* The corresponding author: School of Electrical and Computer Engineering, Georgia Institute of Technology, Atlanta, GA 30332-0250, USA, email: liye@ece.gatech.edu, Phone/Fax: +1 404 385-2256/894-7883 and IV, we develop an antenna selection approach and a power allocation approach, respectively. Finally, we demonstrate performance improvement through computer simulation.

\section{SYSTEM DESCRIPTION}

In this section, we will first introduce wireless propagation model, which will be used in our study and then we describe wireless stadium as an example of hot-spot wireless access.

\section{A. Wireless Propagation Model}

Radio propagation is often very complicated and different in different environments. In general, it can be characterized by three phenomena: path loss, shadowing, and multipath fading. The fading characteristics of distributed antennas have been investigated in [4]. Here we only briefly describe a simplified model that will be used in our study.

Path loss depends on the distance between the transmit and receive antennas. Denote $P_{t}$ and $\bar{P}_{r}$ as the transmitted signal power and the average received signal power, respectively. Pass loss is defined as

$$
L=\frac{\bar{P}_{r}}{P_{t}}
$$

It can be modeled as [7]

$$
L=K \frac{1}{d^{\alpha}},
$$

where $K$ is a constant determined by antenna configuration, $d$ is the distance between the transmit antenna and the receive antenna, and $\alpha$ is called path loss exponent. The pass loss exponent, $\alpha$, is 2 in free space, is usually between 2 and 4, and may be as large as 6 sometimes. 
Due to path loss and shadowing, the actual received signal power can be expressed as

$$
P_{r}(\mathrm{~dB})=\bar{P}_{r}(\mathrm{~dB})+\epsilon(\mathrm{dB}),
$$

where $\epsilon$ represents the effect of shadowing and is a random variable. The shadowing, $\epsilon$, in $\mathrm{dB}$ is often modeled as normal distribution with variance $\sigma_{s}^{2}$. $\sigma_{s}$ is usually between $4 \mathrm{~dB}$ and $10 \mathrm{~dB}$ and its typical value is $8 \mathrm{~dB}$.

Besides path loss and shadowing, radio signal also experiences multipath fading, which causes received signal level changes quickly with time or position of mobile. Consequently, the instantaneous power of the received signal can be expressed as

$$
P=P_{r}|h|^{2}=\beta|h|^{2} P_{t},
$$

where $h$ is a base-band complex channel gain and $\beta=L \epsilon$. For simplicity, the complex channel gain is frequently modeled as complex Gaussian with zero mean and unit variance. As a result, $|h|^{2}$ is with exponential distribution. Furthermore, we assume that $|h|^{2}$ is with unit average.

It should be indicated that both shadowing and multipath fading cause signal power to change with time. However, time-varying due to shadowing is much slower than due to multipath. Therefore, in our study, we assume that shadowing parameter $\epsilon$ can be fed back to the transmitter for performance optimization while instantaneous complex channel gain is not available at the transmitter.

\section{B. Wireless Stadium}

We use wireless stadium as an example in this paper even through the approaches developed here can be directly used to all indoor environments.

As in Figure 1, the stadium is modeled as a circle. The sports are played in the inner circle with a radius of $R=150$ meters. The audience are between the outer and the inner circles and need wireless access so that they can track their favorite players clearly. The radius difference between the two circles is $D=30$ meters.

There are 30 access antennas with 15 uniformly distributed in the outer and the other 15 in the inner circles. The audience area is equally divided into 30 cells, which are marked $C_{n, k}$ for $n=1,2,3$ and $k=1, \cdots, 10$, as in the figure. The available frequency set is equally divided into three different subsets and the 10 cells with the same first index, $n$, use the same frequency subset. Consequently, the same frequency may be used by as many as 10 cells simultaneously. It will cause multi-user

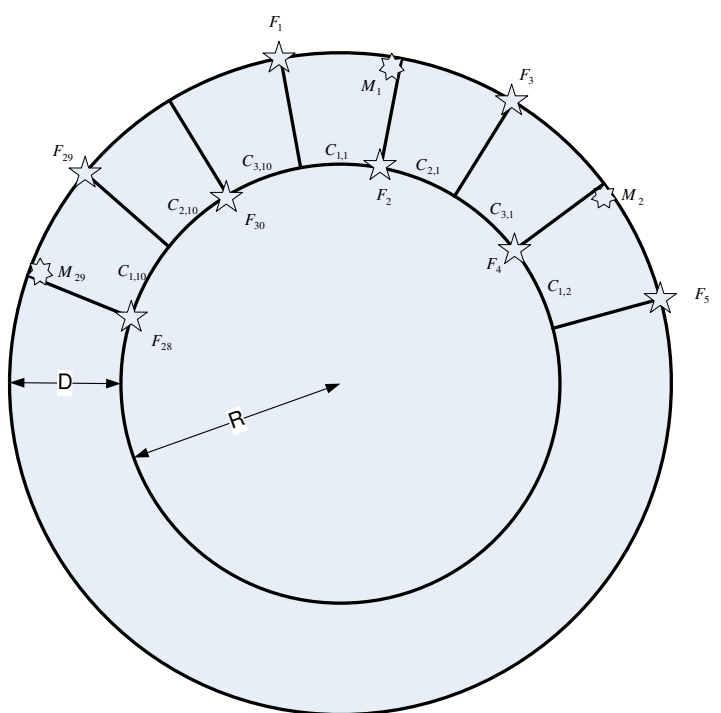

Fixed Antenna for Mobile Access

Antenna for Mobile Device

Fig. 1. Antenna distribution for a stadium.

interference (MUI). In the next section, we will develop approaches to reduce MUI.

Without loss of generality, we only consider 10 cells using the same set of frequency, $C_{1, k}$ 's for $k=1, \cdots 10$, and those users in the edge of these cells, $M_{k}$ 's for $k=1, \cdots 10$, as in Figure 1. Denote $\beta_{m k}$ the power gain that only takes path loss and shadowing into account and corresponds to the $m$ th transmit antenna, $F_{m}$, and the mobile user, $M_{k}$. Then the received signal at $M_{k}$ can be expressed as

$$
y_{k}=\sum_{m \in \mathcal{M}} \sqrt{\beta_{m k}} h_{m k} s_{i_{m}}+n_{k}
$$

where $\mathcal{M}$ is the index set of access antennas used to transmit at the same frequency simultaneously, $h_{m k}$ is the complex channel gain due to multipath fading, $s_{i_{m}}$ is the transmitted signal at access antenna $F_{m}$ for user $i_{m}$, and $n_{k}$ is additive white Gaussian noise (AWGN).

\section{ANTENNA SELECTION}

After introducing a fixed antennas selection approach that considers only pass loss, we develop an optimal antenna selection approach that takes pass loss, shadowing, and interference into consideration.

\section{A. Fixed Antenna Selection}

We can select the access point/anatnna that is the closest to each mobile user, which we call fixed 
antenna selection (FAS). It is obvious that FAS is optimal if there is no shadowing. Consequently, mobile $M_{k}$ will use access point $F_{m_{k}}$, where

$$
m_{k}=\left\{\begin{array}{l}
3(k-1)+2, \text { if } k \text { is odd } \\
3(k-1)+1, \text { if } k \text { is even } .
\end{array}\right.
$$

Therefore, the receive signal at mobile $M_{k}$ can be expressed as

$$
y_{k}=\sqrt{\beta_{m_{k} k}} h_{m_{k} k} s_{k}+\sum_{i \neq k} \sqrt{\beta_{m_{i} k}} h_{m_{i} k} s_{i}+n_{k} .
$$

Consequently, the average signal power and interference-plus-noise power over multipath fading, $h_{m k}$, are

$$
P_{S}=\beta_{m_{k} k} p_{k}
$$

and

$$
P_{I}=\sum_{i \neq k} \beta_{m_{i} k} p_{i}+\sigma_{N}^{2},
$$

respectively, where

$$
p_{k}=E\left|s_{k}\right|^{2} \text {, and } \sigma_{N}^{2}=E\left|n_{k}\right|^{2} .
$$

If all access points transmit signals with the same power, i.e., $p_{i}=P_{t}$ for all $i$ 's, then signal-tointerference-plus-noise ratio will be

$$
\operatorname{SINR}_{k}=\frac{\beta_{m_{k} k} P_{t}}{\sum_{i \neq k} \beta_{m_{i} k} P_{t}+\sigma_{N}^{2}},
$$

the average capacity for FAS will be ${ }^{1}$

$$
C_{\mathrm{FSE}}=E\left\{\log _{2}\left(1+\frac{\beta_{m_{k} k}\left|h_{m_{k} k}\right|^{2} P_{t}}{\sum_{i \neq k} \beta_{m_{i} k}\left|h_{m_{i} k}\right|^{2} P_{t}+\sigma_{N}^{2}}\right)\right\}
$$

Note that in the above expression, the average is over all random variables, $\beta_{m_{i} k}$ 's, consisting of constant pass loss and random shadowing fading, $h_{m_{i} k}$ 's, complex Gaussian random variables with zero mean and unit variance.

\section{B. Optimal Antenna Selection}

As indicated in Section II.A, radio signal will experience three almost independent phenomena: pass loss, shadowing, and multipath fading. The pass loss is fixed and is determined by the distance between the transmit and the receive antennas. The shadowing is random and independent for different points/antennas. Since shadowing changes with time very slowly, shadowing parameters can be

\footnotetext{
${ }^{1}$ We approximate interference as Gaussian to obtain the capacity formula.
}

fed back to the transmitters to exploit shadowing diversity of distributed access antennas.

Due to shadowing, FAS described above is not necessarily optimal. With shadowing parameters, we can select access antenna for each mobile to maximize the SINR. From the above discussion, we should find a mapping from $k$ to $m_{k}$ such that $\mathrm{SINR}_{k}$ in (3) is maximized for all $k$, which turns out to be a complicated non-linear programming problem.

To reduce the complexity of the above problem, we select access antenna for each mobile to maximize signal-to-leakage radio (SLR),

$$
\mathrm{SLR}_{k}=\frac{\beta_{m_{k} k} P_{t}}{\sum_{i \neq k} \beta_{m_{k} i} P_{t}+\sigma_{N}^{2}} .
$$

It should be indicated that optimizing SLR has been used [12], [13] to find optimal steering vector for down-link beamforming of spatial multiplexing systems.

As in Figure 1, the fixed access point, $F_{3}$, is close to mobiles, $M_{1}$ and $M_{2}$, and it is sometimes possible, even through seldom happens, that $F_{3}$ is selected to transmit (different) signals to the two users at the same frequency simultaneously, that is, $m_{1}=m_{2}=3$, which will cause a problem. First, it implies that the fixed access point send different signals to two different mobile users using the same frequency at the same time, which is impossible. Secondly, at least of one of $\mathrm{SINR}_{1}$ and $\mathrm{SINR}_{2}$ is less that 1 , which implies that access point, $F_{3}$, is not good for either of $M_{1}$ and $M_{2}$ and we should exclude it when search for optimal access points for $M_{1}$ and $M_{2}$. To reduced the search complexity, for $M_{1}$, we only compare SLR for two fixed access points, $F_{1}$ and $F_{2}$, that is, $m_{1}=1$ or 2 , depending on which gives a larger SLR. Similarly, we search $F_{4}$ and $F_{5}$ to find an optimal access point for $M_{2}$, and so on.

Once an optimal access point for each mobile, $m_{k}$, is determined, we can find the average capacity using Equation (4).

\section{Power Allocation}

The performance of the system can be further improved by optimally allocating transmission power for each mobile and taking both signal and interference strengthes into consideration.

In this section, we assume the average transmission power for each mobile, $p_{k}$, is $P_{t}$, that is,

$$
\frac{1}{10} \sum_{i=1}^{10} p_{k}=P_{t} \text { with } p_{k} \geq 0, k=1, \cdots, 10 .
$$


Ideally, we should allocate power to maximize the average capacity of all mobiles, that is,

$C=\frac{1}{10} \sum_{k=1}^{10} E\left\{\log _{2}\left(1+\frac{\beta_{m_{k} k}\left|h_{m_{k} k}\right|^{2} p_{k}}{\sum_{i \neq k} \beta_{m_{i} k}\left|h_{m_{i} k}\right|^{2} p_{i}+\sigma_{N}^{2}}\right)\right.$

However, the implementation of the criterion is very complicated since the expectation should be taken over $h_{m_{i}, k}$ 's. Therefore, we come up with a of suboptimal criterion.

Instead of using the average capacity, we use what we call pseudo-capacity (PC) criterion to allocate transmission power, that is, allocate the power to maximize

$$
C_{p c}=\frac{1}{10} \sum_{k=1}^{10} \log _{2}\left(1+\frac{\beta_{m_{k} k} p_{k}}{\sum_{i \neq k} \beta_{m_{i} k} p_{i}+\sigma_{N}^{2}}\right) .
$$

The difference between (6) and (7) is that the expectation is removed in (7) and $\left|h_{m_{i} k}\right|^{2}$ is substituted by its average.

From traditional gradient algorithm, we have the following algorithm to find the optimal power allocation to maximize $C_{p c}$ :

Step (i): Initialization:

$$
\mathbf{p}^{(0)}=\mathbf{1} P_{t} \text {. }
$$

Step (ii): Iteration: for $i=1,2, \cdots$,

$$
\begin{aligned}
& C_{p c}^{(i-1)}=\frac{1}{10} \sum_{k=1}^{10} \log _{2}(1+ \\
& \left.\frac{\beta_{m_{k} k} p_{k}^{(i-1)}}{\sum_{j \neq k} \beta_{m_{j} k} p_{j}^{(i-1)}+\sigma_{N}^{2}}\right) \\
& \Delta \tilde{\mathbf{p}}^{(i)}=\left(\begin{array}{c}
\frac{\partial C_{p c}^{(i-1)}}{\partial p_{1}^{(i-1)}} \\
\vdots \\
\frac{\partial C_{p c}^{(i-1)}}{\partial p_{10}^{(i-1)}}
\end{array}\right) \\
& \boldsymbol{\Delta} \mathbf{p}^{(i)}= \begin{cases}\boldsymbol{\Delta} \tilde{\mathbf{p}}^{(i)}, & \text { if }\left\|\boldsymbol{\Delta} \tilde{\mathbf{p}}^{(i)}\right\| \leq \delta_{1}, \\
\frac{\boldsymbol{\Delta} \tilde{\mathbf{p}}^{(i)}}{\left\|\boldsymbol{\Delta} \tilde{\mathbf{p}}^{(i)}\right\|}, & \text { if }\left\|\boldsymbol{\Delta} \tilde{\mathbf{p}}^{(i)}\right\|>\delta_{1},\end{cases}
\end{aligned}
$$

and

$$
\tilde{\mathbf{p}}^{(i)}=\tilde{\mathbf{p}}^{(i-1)}+\mu \Delta \mathbf{p}^{(i)} .
$$

Step (iii): Checking Constraint:

$$
\hat{p}_{k}^{(i)}= \begin{cases}\tilde{p}_{k}^{(i)}, & \text { if } \tilde{p}_{k}^{(i)}>\delta_{2}, \\ \delta_{2}, & \text { if } \tilde{p}_{k}^{(i)} \leq \delta_{2}\end{cases}
$$



Fig. 2. Performance versus the normalized transmission power when $\alpha=3.5$ and $\sigma_{s}=8 \mathrm{~dB}$.

for $k=1, \cdots 10$, and

$$
\mathbf{p}^{(i)}=\frac{P_{t}}{\operatorname{mean}\left(\hat{\mathbf{p}}^{(i)}\right)} \hat{\mathbf{p}}^{(i)} .
$$

Step (iv): Repeating Steps (ii) and (iii) if \| $\mathbf{p}^{(i)}-\mathbf{p}^{(i-1)} \| \leq \delta_{3}$.

In the above $\delta_{1}, \delta_{2}$, and $\delta_{3}$ are small positive numbers and $\mu$ is called step size..

\section{Simulation Results}

To demonstrate the performance of the proposed approaches, computer simulation is performed. In all of our simulation, the transmission power per $\mathrm{Hz}$ is normalized by the power spectral density of noise. When calculating the average capacity, the fast fading due to multipath also takes into consideration.

Figure 2 shows the average capacity versus transmission power for different algorithms when the path loss exponent of channel is $\alpha=3.5$ and the standard deviation of shadowing is $\sigma_{s}=$ $8 \mathrm{~dB}$. From the figure, optimal antenna selection can significantly improve the system performance compared with fixed antenna selection. Without power allocation, optimal antenna selection can increase the throughput by about $30 \%$ when the normalized transmission power, $P_{t}=120 \mathrm{~dB}$. The pseudo-capacity based power allocation can further improve the network throughput, especially when there is no antenna selection.

Figures 3 (a) and (b) show the average capacity versus the path loss exponent and versus shadowing, respectively. From Figure 3 (a), the network 

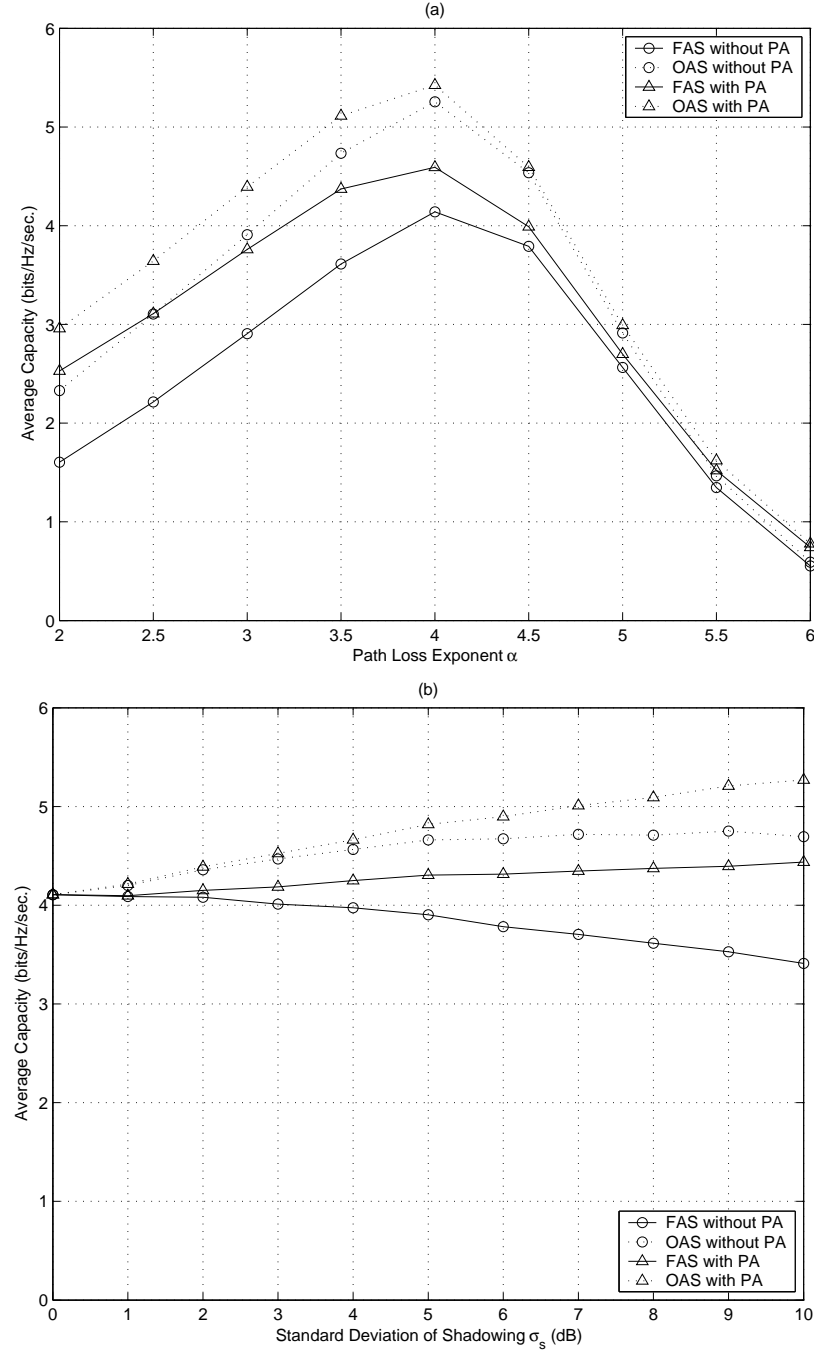

Fig. 3. Performance versus (a) the path loss exponent when $P_{t}=120 \mathrm{~dB}$ and $\sigma_{s}=8 \mathrm{~dB}$ and (b) the standard deviation of shadowing when $P_{t}=120 \mathrm{~dB}$ and $\alpha=3.5$.

reaches the best performance for the environment with a pass loss exponent of around 4.0. From Figure 3 (b), the optimal antenna selection can improve the network performance and the performance improvement increases with the standard deviation of shadowing.

\section{CONCLUSIONS}

In this paper, we have described our research results in antenna selection and power allocation for hot-spot communications. We have developed an optimal antenna selection approach, which takes both desired signal strength and interference strength into consideration when selecting antennas. We have also proposed a power allocation approach that can significantly improve the average capacity of the network. Even through we only use a wireless stadium as an example in the paper. The developed techniques can be directly used to other indoor environments.

\section{REFERENCES}

[1] A. A. M. Saleh, A. J. Rustako, Jr., and R. S. Roman, "Distributed antennas for indoor radio communications," IEEE Trans. Commun., vol. 35, pp. 1245-1251, Dec. 1987.

[2] L. J. Greenstein, N. Amitay, T.-S. Chu, L. J. Cimini, Jr., G. J. Foschini, M. J. Gans, C.-L. I, A. J. Rustako, Jr., R. A. Valenzuela, and G. Vannucci, "Microcells in personal communications systems," IEEE Commun. Mag., pp. 76-88, Dec. 1992.

[3] G. J. Pottie, "System design choices in personal communications," IEEE Personal Commun., pp. 50-67, Oct. 1995.

[4] K. W. Cheung and R. D. Murch, "Fading characteristics of distributed antennas," Electronics Letters, vol. 32, no. 3, pp. 172-174, Feb. 1996.

[5] K. J. Kerpez, "A radio access system with distributed antennas," IEEE Trans. Commun., vol. 45, pp. 265-275, May 1996.

[6] H. H. Xia, A. B. Herrera, S. Kim, and F. S. Rico, "A CDMA-distributed antenna system for in-building personal communications service," IEEE J. Sel. Areas Commun., vol. 14, pp. 644-650, May 1996.

[7] Y. (G.) Li and G. L. Stüber, OFDM for Wireless Communications, Springer, 2006.

[8] L. Dai, S. Zhou, and Y. Yao, "Capacity analysis in CDMA distributed antenna systems," IEEE Trans. Wireless Commun., vol. 4, pp. 2613-2620, Nov. 2005.

[9] V. Nikolopoulos, M. Fiacco, S. Stavrou, and S. R. Saunders, "Narrowband fading analysis of indoor distribted antenna systems," IEEE Ant. and Wireless Prop. Lett., vol. 2, pp. 89-92, 2003.

[10] A. Ettefagh, M. Kuhn, B. Cheetham, and A. Wittneben, "Comparison of distributed and co-located antenna diversity schemes for the coverage improvement of VoWLAN systems," Proc. IEEE PIMRC'05, Berlin, Germany, Sept. 2005, pp. 77-81.

[11] S. Han, S. Zhou, J. Wang, V. O. K. Li, and K. Park, "Suboptimal transmission of orthogonal space-time block codes over correlated distributed antennas," IEEE Trans. on Signal Processing, vol. 14, pp. 89-92, Feb. 2007.

[12] Y. (G.) Li, A. C. K. Soong, Y.-G. Du, and J.-M. Lu, "Beamforming with imperfect CSI," Proc. IEEE Wireless Commun. and Networking Conf., Hong Kong, China, March 2007.

[13] A. Tarighat, M. Sadek, and A. H. Sayed, "A multi user beamforming scheme for downlink MIMO channels based on maximizing signal-to-leakage ratios," Proc. of IEEE International Conf. on Acoustics, Speech, and Signal Processing (ICASSP'05), pp. 18-23, March 2005. 